\title{
Congresos nacionales de pediatría 1994-1995. Una comparación del área de temas libres
}

\author{
Gastón Duffau T.'
}

\begin{abstract}
Resumen
La rev sión de los resúmenes que posiulcron en 1994 y 1995 a los congresos ncciona es de peciatria muesiro que la moyer parte de los rabajos preseniados procedían de Saniago. En 1995 hupo un descenso nolable en la parlícipación de regiones. Sólo 4 a 5 de las postulaciones procedía de olros países. Lo proporción de trabajos cceptodos no varió en los dos periodos para Saniago y regiones, pero mejóó paro os extranieros. En regiones sólo 15 localidades postularon an 1994 y 14 en 1995, coincidiencio 10 en ombos perícdos. las áreas preferidcs de estudio er Santiago fueron, en ambos cños, riufriciór. salud pública y reurología. En cambio los autores regiona es péiriercn carciolog'a, gostroenlerolocía y nefrología los resúmenes con mós probat. lidades de ser aceptocos fuercn los de diseño prospectivo, con los zinco párrabos solizi-ados jintraducción, hipónesis, malerial y método, resultados y conclusianies?, con conclusiones explicitas y efectivamente derivades de los resultados. Las instituciones privadas de solud uvieron escasa participación. Se requieren estimulos a la investigación en consultorios externos y regiones y a la 'ncorporación de mcyor número de localidades a astc actividad
\end{abstract}

'Palabras clave: investigación, comunicación, congresos, conferencias.'

\section{Trends on free reports to chilean pediatric congresses}

A review of abstrccls submitted 10 1994 and $1995 \mathrm{C}$-ilean National Congresses of Pedialrics is presenled. Most aostracis were submilted by institutions from Santiggo, the capital cily of the country. There was a clear decrease. ro $58 \%$ of pecedent year, in the proportion of abslacts irom the rest of the couniry in 1995. Contributions from ather countries did roi change and ranged from 4 to $5 \%$ of the toal. The poportion of accepled abstracis was similar in 1994 and 1995: with the exceptiar of those from abrood. Absiracls of authors from 15 eities other than Santiago we submitted i- 1994 and 14 in 1995, 10 places being represented in both yocrs. Most frequent repon subjects for investigotors from Sonficgo ware rutrilion, public regl.h and neurology, while cardiology, gostroenterology and nephrology were the prefered areas for parlicipants rom other ioca ities. Abstracts with higher chances for beirig accepted were those of prospective studies including an introduction, hypothesis, matherial \& methocs, results and conclussions]. with explici coriclussions clealy sustained by sesults. Contribution of members of privale health institutions were sca ce. Slimu:us to reseprer. are ai need ir culpotients clinics and in regioncl health centers far from the metropolian area of the capital city.

Key words: reseorch, reports, conterences, congresses.

Los congresos nacionales de pediatría represcntan la oportunidad donde quienes cultivan la especiaidad madre y sus derivaciones, pueden encontrarse y compartir sus experiencias. La exposición de temas libres ofrece todas las opciones, con la única exigencia de que se respeten

I. Departamento de Pediatria y Cirugia Infantit, Campus Norte. Facultad de Medicina Universidad de Chile. Hospital Clínico de Niños Roberto del Rio. las normas de postulación, formato $\mathrm{y}$ fondo metodológico que es propio aplicar en estas actividades.

La descripción de las postulaciones al área de temas libres y su evolución permitirá detectar características y cambios favorables o desfavorables que promuevan medidas de estímulo para lo primero y de corrección, si es posible, para lo segundo. Tal es el sentido de la presentación que sigue, apoyados en el planteamiento que no 
ha cambiado la forma de evaluar las postulaciones en el período analizado. De hecho así ha sido, puesto que se ha mantenido el mismo instrumento y esquema de puntaje, objetivo hasta donde es posible, niveles de aceptación/rechazo, básicamente los mismos evaluadores y dirección del proceso.

\section{Material y Método}

Se dispuso de la totalidad de postulaciones a los congresos nacionales de 1994 y 1995. Para el primero se recibieron 218 resúmenes y para el segundo 176. Todos habían sido analizados por las comisiones científicas respectivas, empleando el mismo instrumento, asignando a cada uno un puntaje de hasta 95 puntos. La separación entre "aceptados" y "techazados" se realizó de acuerdo a criterio descrito en otra parte".

Se respeto la recomendación de los autores para elegir el área temática a la que pertenecía el estudio. Tanto en el caso de indicar más de una o ninguna, se decidió por aquella que arbitrariamente pareció más plausible. De cada resumen se extrajo la siguiente información: lugar geográfico de origen, área temática, diseño declarado por los autores o asignado posrevisión. número de autores participantes, existencia de cinco acápites o párrafos exigidos en las instrucciones para postular (introducción; hjpótesis u objetivos; material y método; resultados: conclusiones), presencia de conclusiones explícitas, financiamiento, institución de origen (1995).

En la estadistica se recurrí́ a pruebas de $\mathrm{Ji}^{2}$ con corrección de Yales. Fisher-Irwin de proba- bilidades exactas y comparación de porcentajes $^{2}$. Se aceptó un error alfa de $5 \%$.

\section{Resultados y Comentario}

En la tabla 1 se comparan los dos conjuntos de postulaciones de acuerdo a su procedencia. En ambos es notable el predominio porcentual de Santiago sobre las regjones, cuya participación disminuyó significativamente en 1995 ( $p<0,05$ ). La proporción de trabajos extranjeros fue similar en ambos períodos, de 4 a $5 \%$. En regiones, la disminución de postulaciones en términos absolutos fue importante, ya que en 1995 constituyeron el $58,4 \%$ de aquétlas del año anterior. En Santiago, en cambio, la variación mostró caída a sálo $93,2 \%$ del volumen correspondiente a 1994.

En la misma tabla es posible ver que aplicando en los dos años analizados el mismo criterio, la proporción de aceptados en 1994 vs. 1995 fue para Santiago $78,0 \%$ y $77,2 \%$ respectivamente, referidos al total procedente de esta ciudad y $47.2 \%$ y $53.9 \%$ respectivamente, referidos al total general de resúmenes. Ningún cambio fue significativo ( $p>0,05)$, situación que se mantiene aun retirando los trabajos extranjeros. $\mathrm{La}$ proporción de estos últimos, que fueron aceptados respecto al total general, aumentó significativamente: $\mathrm{Ji}^{2}$ con corrección de Yates $=4,42$ y $\mathrm{p}<0,05$. Comparando sólo resúmenes extranjeros entre sí ocurre algo similar: por Fisher-Irwin $\mathrm{p}=0.003$. En regiones se aceptó $74,0 \%$ vs. $75,5 \%$ de las postulaciones de 1994 vs. 1995. porcentajes que sobre el total general fueron dé $26,1 \%$ vs. 19,3\%. En ambos casos p >0,05. Asi-

\section{Tabla 1}

Lugar de origen y resultado de la postulación en dos congresos nacionales de Pediatria

\begin{tabular}{|c|c|c|c|c|c|c|c|}
\hline \multirow{3}{*}{ Sintiago } & \multicolumn{3}{|c|}{1994} & \multicolumn{4}{|c|}{1995} \\
\hline & \multirow{2}{*}{ Aceptados } & \multirow{2}{*}{$\frac{\text { Rechazados }}{29}$} & Total & Aceptados & Rechazados & \multicolumn{2}{|c|}{ Total } \\
\hline & & & $1.32(60.6)^{*}$ & 95 & 28 & 123 & $(69,9)^{* *}$ \\
\hline Regiones & 57 & 20 & $77(3,5,3)^{*} \#$ & 34 & 11 & 45 & $(25,6)^{* * * \#}$ \\
\hline Extranjeros & I & 8 & $9(4,1)$ & 7 & 1 & 8 & $(4.5)$ \\
\hline Toral & 161 & 57 & $218(100)$ & I 36 & 40 & 176 & $(100)$ \\
\hline
\end{tabular}

*. ${ }^{* *}, \# p<0,05$ 
mismo, es interesante señalar que en 1994 postularon 15 localidades y 14 en 1995, haciéndolo 10 en ambos años (Antofagasta, Concepción, Coquimbo, Coronel, Iquique, Valdivia, Valparaíso, Viña del Mar, Talcahuano, y Temuco). Estas localidades presentaron en el períndo un total de 99 resúmenes, logrando la aceptación de $77(77,7 \%)$ y las restantes, $14 / 23(60,9 \%)$ $(\mathrm{p}>0.05)$.

Las áreas temáticas de investigación preferidas según región geográfica se muestran en la tabla 2. La razón Santiago/regiones, de número de trabajos, cambió de $1,71 / 1$ en 1994 a 2,57/1 en 1995. Los temas preferidos en Santiago, respecto a regiones, considerando así aquellos casos en que la razón particular supera el valor general del año fueron adolescencia, inmunología, nutrición pediatría general y ambulatoria, salud pública y neurología en 1994 y, en 1995 , broncopulmonares, infectología y nutrición, tratamiento intensivo, salud pública y neonatología-neurologia.

En regiones destacaron cardiologia, gastroenterología y nefrología. Como sea, es llamativo que no predominen notoriamente en regiones sobre Santiago los estudios de pediatría general y ambulatoria o los de salud pública que, en principio, ofrecen más probabilidad de permitir trabajos con menos apoyo de laboratorio. Ahora, en la distribución de los trabajos por temas es necesario considerar que algunas especialidades derivadas se han alejado en forma manifiesta de la especialidad madre $y$, entonces, participan principalmente en sus propias jornadas $y$ congresos.

En la tabla 3 se muestra el diseño de los estudios según aceptación o rechazo. En esencia, los resúmenes postulantes podían ser catalogados como estudios prospectivos, retrospectivos, casos clínicos y un conjunto de "otros". Los estudios prospectivos corresponden básicamente a ensayos clínicos y seguimiento de pacientes; los retrospectivos a descripción de series clínicas que son consideradas de interés. No se encontró ningún estudio caso-control (aunque si de casos y controles). El rubro "otros" incluye programas de atención, rutinas para computación, descripciones de métodos, etc.

Al comparar los diseños aceptados y rechazados en los años en análisis, es posible encontrar

Tabla 2

Areas preferidas de investigación. Santiago vs. regiones*

\begin{tabular}{|c|c|c|c|c|c|c|}
\hline \multirow[t]{2}{*}{ Area temática } & \multirow{2}{*}{$\begin{array}{c}\text { Santiago } \\
1994\end{array}$} & \multirow{2}{*}{$\begin{array}{c}\text { Regiones } \\
1995\end{array}$} & \multicolumn{2}{|c|}{ Razón } & \multicolumn{2}{|c|}{ Santiago/Reg. } \\
\hline & & & 1994 & 1995 & 1994 & 1995 \\
\hline Adolescencia & 7 & 2 & 0 & 0 & $7,00 \div 0$ & $2,00: 0$ \\
\hline Broncopulınonares & 20 & 11 & 12 & 2 & $1.66: 1$ & $5,50: 1$ \\
\hline Cardiología & 2 & 5 & 5 & 3 & $0.40: 1$ & I,66:1 \\
\hline Endocrino-Genética & 14 & 7 & 9 & 3 & $1.55: 1$ & $2,33: 1$ \\
\hline Gistroenterología & 10 & 9 & 8 & 4 & $1,25: 1$ & $2,25: 1$ \\
\hline In fectología & 7 & 8 & 7 & 2 & $1,00: 1$ & $4,00: 1$ \\
\hline Inmunologiáa & 6 & 0 & 0 & 1 & $6.00: 1$ & $0,00: 1$ \\
\hline Maltmo infantit & 5 & 0 & 3 & 0 & $1,66: 1$ & $0.00: 0$ \\
\hline Nefrología & 6 & l & 7 & 4 & $0,85: 1$ & $0,25: 1$ \\
\hline Neonatología & 20 & 27 & 12 & 7 & $1,66: 1$ & $3,85: 5$ \\
\hline Nutrición & 5 & 9 & l & 2 & $5,00 \div 1$ & $4,50: 1$ \\
\hline Pedialría Gral. y Ambulatoria & 11 & 10 & 4 & 4 & $2,75: 1$ & $2,50: 1$ \\
\hline Salud Pública & 7 & 16 & 2 & 5 & $3,50: 1$ & $3.20: 1$ \\
\hline Tratamiento Intensivo & 4 & 6 & 3 & 2 & $1,33: 1$ & $3,00: 1$ \\
\hline Neurología & 4 & 5 & 2 & 1 & $2,00: 1$ & $5,00: 1$ \\
\hline Reumatología y Oncología & 3 & 2 & 2 & 1 & $1,50: 1$ & $2,00: 1$ \\
\hline Radiologia & 1 & 0 & 0 & 0 & $1,00: 0$ & $0,00: 0$ \\
\hline Psiquiatría & 0 & 2 & 0 & 6 & $0,00: 0$ & $0.33: 1$ \\
\hline Parasitologia & 0 & $\mathbf{I}$ & 0 & 0 & $0,00: 0$ & $1,00: 0$ \\
\hline Total & 132 & 121 & 77 & 47 & $1,71: 1$ & $2,57: 1$ \\
\hline
\end{tabular}

* No están incluidos 9 1rabajos extranjeros en 1994 y 8 en 1995. 
Tabla 3

Tipo de estudio y resultado de la postulación en dos congresos Nacionales de Pediatría

\begin{tabular}{|c|c|c|c|c|c|c|c|c|c|c|c|}
\hline Aceptados & Año & \multicolumn{2}{|c|}{ Prospectivos } & \multicolumn{2}{|c|}{ Retrospectivos } & \multicolumn{2}{|c|}{ Casos clínicos } & \multicolumn{2}{|c|}{ Otros } & \multicolumn{2}{|c|}{ Total } \\
\hline & 1994 & 73 & $(45,4)$ & 72 & $(44.7)$ & 16 & $(9,9)$ & 0 & $(0,00) \#$ & 161 & $(100)$ \\
\hline & 1995 & 66 & $(48,5)$ & 49 & $(36,1)$ & 10 & $(7,3)$ & 11 & $(8,1)^{\#}$ & 136 & $(100)$ \\
\hline \multicolumn{12}{|l|}{ Rechazados } \\
\hline & 1994 & 1 & $(1.7)^{* *}$ & 23 & $(40,4)$ & 17 & $(29.8)$ & 16 & $(2 B, 1)^{*}$ & 57 & $(100)$ \\
\hline & 1995 & 7 & $(17,5)^{* *}$ & 19 & $(47.5)$ & 10 & $\{25,0)$ & 4 & $(10,0)^{*}$ & 40 & $(100)$ \\
\hline
\end{tabular}

$\# p=0,0007 \quad * * p=0,007 \quad * p=0.01$.

que la única diferencia significativa en los aceptados la da el rubro "otros", que aumentó de 0\% a $8,1 \%$. En 1995 hubo un incremento importante del rechazo de estudios prospectivos $y$ en cambio una disminución en "otros".

Según número de autores participantes es interesante destacar que en 1995 se privilegió la aceptación de estudios en que participó un grupo mayor de investigadores. Asi, la proporción de aceptados con 5 o más autores se elevo de $25,2 \%$ (1994) a $39,0 \%(p=0,003)$. Esto podría tener relación con el creciente interés que se observa en nuestro medio por los estudios de grupos, así como por aquellos cooperativos.

En ambos períodos analizados, quienes cumplieron con el requisito de incluir en el resumen los cinco pártafos tuvieron mucho más probabilidades de lograr aceptación de su propuesta: $100 \%$ vs. $58,4 \%$ en 1994 y $92,1 \%$ vs. $66,0 \%$ en 1995 ( $p<0,001$ en ambos casos).

La existencia de conclusiones explícitas fue definitivamente más común en los trabajos aceptados: $65.2 \%$ vs. $3,5 \%$ ( $p<0,05$ ) en 1994 y $72,0 \%$ vs. $52,5 \%$ (p< $<0,05$ ) en 1995 . aunque en este último año aumentaron los rechazados que salisfacian el requisito mencionado. Al menos en parte esto corresponde a conclusiones que, aunque presentes, no se derivan claramenle de los resultados o, lo que a veces es peor, no se requería efectuar el estudio para concluir lo expresado.

El financiamiento obtenido de instituciones que se dedican a ello estuvo presente en proporción reducida de los estudios: $10,0 \%$ en $1994 \mathrm{y}$
$10,2 \%$ en 1995. Esta característica hizo menos probable el rechazo en $1994(9,1 \%$ vs. $28,0 \%$, $\mathrm{p}<0,05)$ pero no en el año siguiente $(11,1 \% \mathrm{vs}$. $24,1 \%, p>0,05)$. Esto deriva, en algunos casos, de presentaciones que no corresponden al estudio principal sino más bien a uno secundario o derivado, que es atribuido al mismo financiamiento.

En 1995 las instituciones declaradas como origen de las investigaciones incluyeron: 24 hospitales (12 de Santiago), 7 universidades, 5 institutos de salud, 3 clínicas privadas, 2 servicios de salud, 2 consultorios externos y el Ministerio de Salud. Las entidades privadas de salud, aisladamente, contribuyeron con $2,5 \%$ de los rechazos y $2,9 \%$ de los resúmenes aceptados en 1995.

Es claro que se requiere estimular la investigación en consultorios externos y en regiones, así como tambián la participación de un gran conjunto de localidades que usualmente no lo hacen. Como parte de tal intención, la Sociedad Chilena de Pediatría creó para 1996 ocho estímulos con el fin de otorgarlos a igual número de investigaciones que postularan al XXXVII Congreso Nacional.

\section{Referencias}

1. Duffau $G$ : Investigación en la Sociedad Chilena de Pediatria. Rev Chil Pediatr 1995; 66: 238-240.

2. Dawson-Saunders B. Trapp R; Bioestadística médica. Edjtorial El Manual Moderno S.A. México D.F. 1993; 73-184. 